\title{
A new species of planarian flatworm from Mexico: Girardia guanajuatiensis
}

\author{
Elizabeth M. Duncan ${ }^{1 \dagger}$, Stephanie H. Nowotarski ${ }^{2,5 \dagger}$, Carlos Guerrero-Hernández ${ }^{2}$, Eric J. Ross ${ }^{2,5}$, \\ Julia A. D'Orazio ${ }^{1}$, Clubes de Ciencia México Workshop for Developmental Biology ${ }^{3}$, Sean \\ McKinney ${ }^{2}$, Longhua $\mathrm{Guo}^{4}$, Alejandro Sánchez Alvarado ${ }^{2,5^{*}}$ \\ ${ }^{\dagger}$ Equal contributors. \\ ${ }^{1}$ University of Kentucky, Lexington KY, USA. \\ ${ }^{2}$ Stowers Institute for Medical Research, Kansas City MO, USA. \\ ${ }^{3}$ Clubes de Ciencia México, Guanajuato, GT, México. \\ ${ }^{4}$ University of California, Los Angeles CA, USA \\ ${ }^{5}$ Howard Hughes Medical Institute, Kansas City MO, USA.
}

\section{Keywords}

planarian, Girardia, Mexico, regeneration, stem cells

\section{ABSTRACT}

\section{Background}

Planarian flatworms are best known for their impressive regenerative capacity, yet this trait varies across species. In addition, planarians have other features that share morphology and function with the tissues of many other animals, including an outer mucociliary epithelium that drives planarian locomotion and is very similar to the epithelial linings of the human lung and oviduct. Planarians occupy a broad range of ecological habitats and are known to be sensitive to changes in their environment. Yet, despite their potential to provide valuable insight to many different fields, very few planarian species have been developed as laboratory models for mechanism-based research.

\section{Results}

Here we describe a previously undocumented planarian species, Girardia guanajuatiensis (G.gua). After collecting this species from a freshwater habitat in central Mexico, we characterized it at the morphological, cellular, and molecular level. We show that G.gua shares features with animals in the Girardia genus but also possesses traits that appear unique to this species.

\section{Conclusion}

Species comparisons are a powerful tool for dissecting both genotype to phenotype and ecology and evolution relationships. By thoroughly characterizing a new planarian species, our work facilitates future species comparisons as well as the molecular dissection of specific G.gua traits.

\section{BACKGROUND}

Biologists have been fascinated by planarian flatworms for centuries, in large part because of their remarkable ability to regenerate missing tissues and body parts (1). Hundreds of diverse planarian species have been identified in numerous locations across the globe and in various environments, including freshwater (2), marine (3), and terrestrial habitats (4). Despite their morphological and ecological diversity, most planarians have at least some regenerative capacity, although there is a wide range of species-specific abilities. At one extreme, some freshwater planarians can recreate an entirely new animal from a small fragment of amputated tissue (5); at the other end of the spectrum, many marine species exhibit very limited regeneration e.g., only within a restricted anatomical region $(6,7)$. This variation in regenerative abilities and ecological 
habitats not only makes planarians an excellent model for studying the cellular mechanisms underlying animal regeneration, but also provides an opportunity to assess the impact of various environmental factors on this complex developmental and reproductive process. For example, excess amounts of industrial pollutants such as ferric iron and organophosphorus pesticides have been shown to affect functional head regeneration (8-10). Such studies provide valuable insight into how changes in the environment, including increased exposure to specific chemicals, can impact both the immediate health of local animals and their ability to maintain their population.

Although their impressive regenerative capacity sets them apart, planarians also bear several biological features that are morphologically and functionally analogous to those of other animals, including humans. One important such feature is the planarian epidermis, which comprises a monostratified mucociliary epithelium that is highly similar to the epithelia of human lungs and oviducts (11). Planarians also possess primitive nephron units, or protonephridia, which share striking structural, functional, and transcriptional identity with their counterparts in human kidneys (12). In short, planarians are a powerful, tractable, in vivo research organism for studying many fundamental cellular structures, functions, and processes.

Given the diversity of planarian species and their differences in regenerative capacity, morphological features, and interactions with the local environments, comparative studies offer enormous potential for uncovering the genomic and cellular mechanisms underlying these unique characteristics. However, only a few planarian species, mainly Schmidtea mediterranea and Dugesia japonica, have been sufficiently developed into models with established tools and infrastructure for such analyses. In order to expand the power of comparative approaches and the number of distinct biological features that can be compared and dissected, we need to identify and thoroughly characterize new planarian species.

Here we describe a newly isolated species that we name Girardia guanajuatiensis (G.gua). We characterize its basic anatomy and capacity for regeneration and describe features of its outer epithelium and stem cell population that distinguish G.gua from other species. We also assembled a G.gua transcriptome, which will greatly facilitate gene expression analyses and forward genetic experiments in future studies. This thorough characterization of Girardia guanajuatiensis will both stimulate further investigations into its intriguing species-specific features and encourage the discovery of more planarian species.

\section{RESULTS}

\section{Animal Collection}

Undergraduate students of a Developmental Biology Workshop organized by Clubes de Ciencia (CdeC) (https://www.clubesdeciencia.mx/) hosted at the University of Guanajuato in Guanajuato, México, embarked on a field expedition to find and study native planarians. We visited the Parque Bicentenario in the southeastern part of Guanajuato City, Mexico to prospect for animals (Figure 1A, red arrowhead). The Parque Bicentenario is home to the Presa San Renovato (Saint Renovato Dam), which controls the flow of several local springs and small rivers. We found the new species in a shallow pool of freshwater with minimal flow opposite the dam (Figure 1B). We observed an aggregation of approximately 300 animals in this pool (Figure 1C) and, upon closer inspection, noted that the majority of animals appeared to be around 1 centimeter in length with dark pigmentation (Figure 1D). We collected approximately 60 animals and transported them back to the laboratory for further characterization (Figures 2-7). The proposed species name, guanajuatiensis, pays tribute to the location of their discovery. 

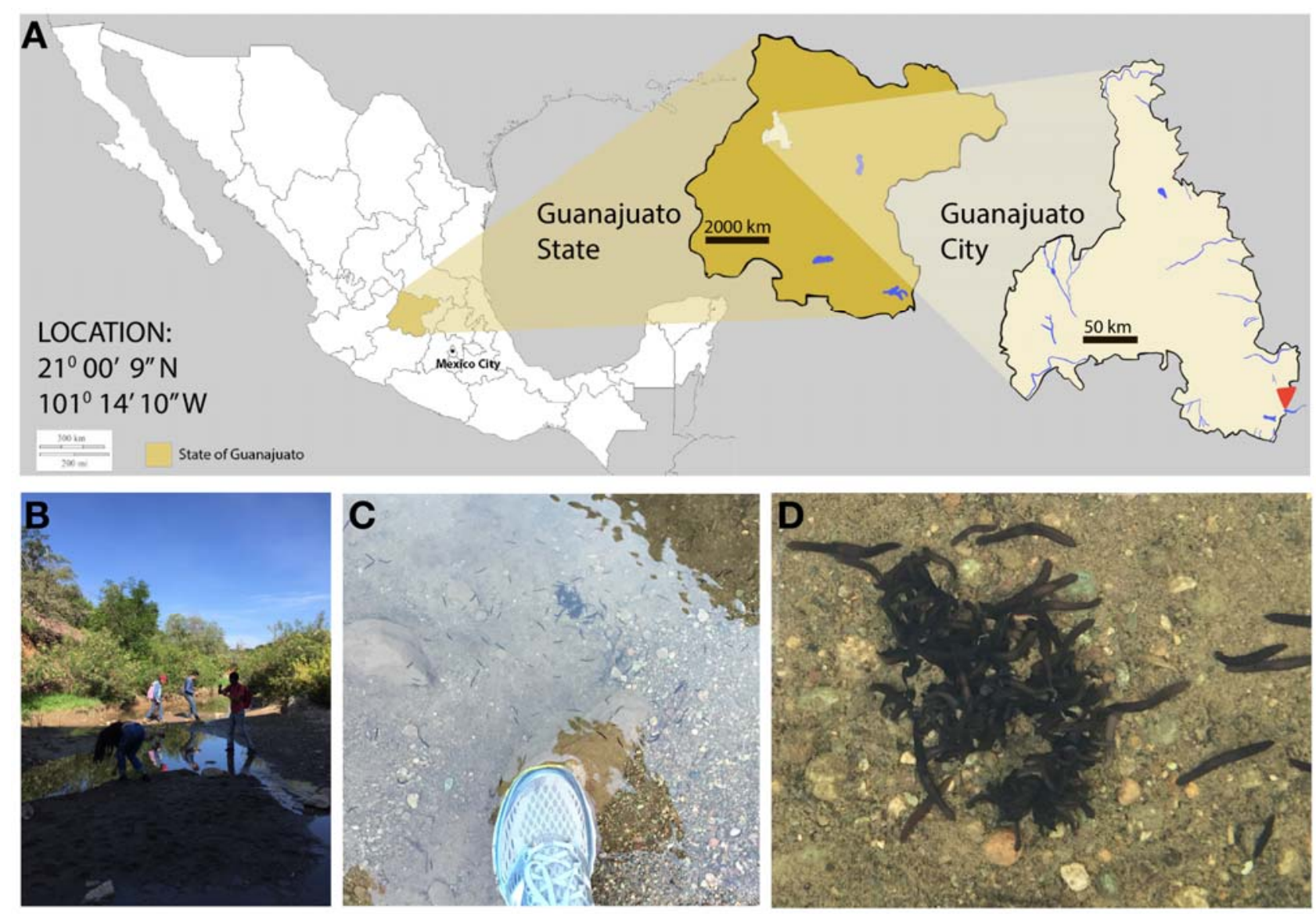

Figure 1. A previously uncharacterized planarian species was collected in Mexico.

(A) A map showing the location of the Presa San Renovato (red arrowhead), a dam within the Parque Bicentenario where the newly named species of planarians was collected. Magnified images show the site is located at the southeastern edge of Guanajuato City in the state of Guanajuato, Mexico. (B-D) The newly named planarian species, G. guanajuatiensis (G.gua), was found at the bottom of a shallow pool of freshwater near the dam.

\section{Morphological Characterization}

In order to assign this guanajuatiensis planarian to a genus, we first characterized their external morphology at low resolution (Figure 2). We immediately noted the presence of two prominent eyespots (or photoreceptors), a triangular head with pointed auricles, and an oblong dorsal patch of lightly-pigmented tissue that is indicative of an internal pharynx (Figure 2A). Upon inspection at higher magnification, we observed that the auricles are densely ciliated (Figure 2B, brackets) with highly motile cilia (Supplemental Movie S1). Several genera of freshwater planarians feature pronounced, ciliated auricles, including Dugesia, Phagocata and Girardia (13-15). Yet the pointed shape of the guanajuatiensis head and auricles largely exclude Phagocata as a possibility. To distinguish between Dugesia and Girardia, we presented animals with food and monitored as they extruded their pharynges to eat. In all worms, we observed a single, pigmented pharynx (Figure 2C). As all other known planarian species with pigmented pharynges are classified as Girardia and this trait is considered apomorphic for the genus (16), this new species likely belongs to the Girardia genus. This assignment is also supported by the fact that only four genera are documented to be endemic to the Neotropical region: Romankenkius, Opisthobursa, Rhodax and Girardia, with Girardia species accounting for the majority (17). 


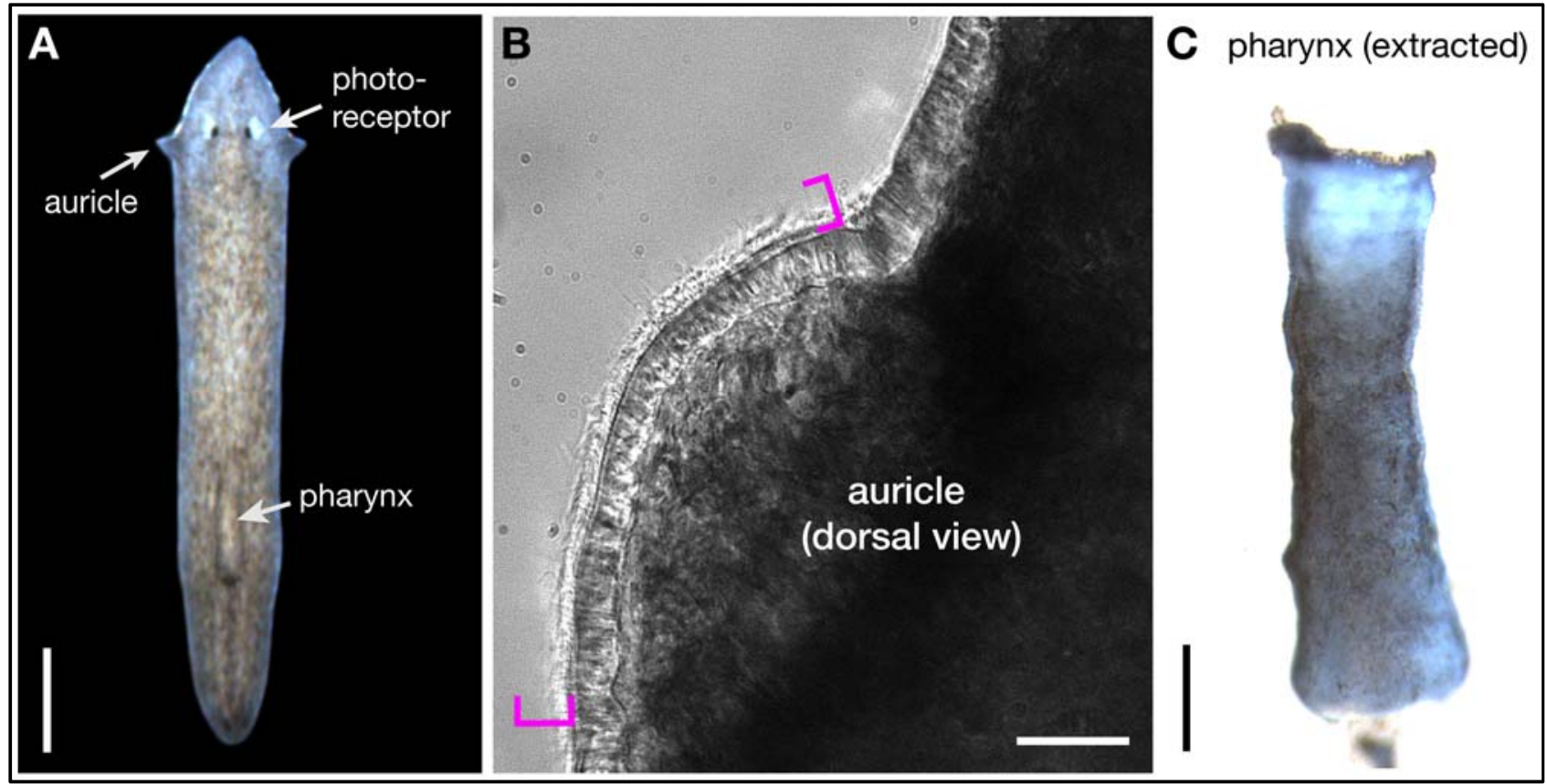

Figure 2. G.gua exhibit standard morphological features associated with Girardia planarians.

(A) Live image of a single G.gua worm after transporting them back to the laboratory. As with other planarians in the Girardia genus, G.gua has prominent, ciliated auricles $(A, B)$ and a pigmented pharynx (C). Scale bars $=500 \mu \mathrm{M}$ in $\mathrm{A}$, $20 \mu \mathrm{M}$ in $\mathrm{B}, 200 \mu \mathrm{M}$ in $\mathrm{C}$. Brackets in (B) indicate cilia projecting from the epidermal cells that cover the auricle.

Planarian locomotion is driven by the concerted beating of motile cilia that protrude from cells in the ventral epidermis $(14,18)$. When we watched the putative Girardia guanajuatiensis (G.gua) migrate towards food in the laboratory, we noticed they frequently performed a peristalticlike motion (Supplemental Movie S2) rather than the canonical smooth glide associated with other common laboratory species. As this motion is reminiscent of the inch-worming phenotype exhibited by other planarian species after RNAi depletion of essential cilia proteins $(19,20)$, we decided to examine the outer mucociliary epidermis of these animals. To characterize all potentially important exterior features of this tissue, we used scanning electron microscopy (SEM) to capture images with detailed resolution. SEM revealed that the dorsal epidermis of G.gua has two distinct regions: 1 ) the auricles, which are densely covered by multiciliated cells (Figure 3A, B, D); and 2) the remaining dorsal surface, which is non-ciliated except for single, sparsely-distributed, ciliated cells that appear in lateral stripes (Figure 3B, C). Examination of the ventral surface by SEM (Figure 3E$\mathrm{H})$ revealed clustered patches of multiciliated cells that span the entire ventral epidermis, although with higher density at the anterior end. In some regions of the animal, these patches are organized in a distinct checkered pattern of single ciliated and non-ciliated cells (Figure 3G). Although this unique pattern of ventral cilia does correlate with and possibly explains the motility differences observed for G.gua animals, we were still surprised by this finding. Most other planarian species, including Schmidtea mediterranea (S.med) and Girardia tigrina $(11,14)$, maintain a dense and apparently uniform distribution of multiciliated cells across their ventral epithelia such that it resembles a lawn of cilia rather than patches.

While maintaining G.gua in the lab, we observed multiple instances of reproduction by fission, which is well documented in the asexual S.med species (21) but rarely seen in the sexual strain of S.med or other sexual planarian species. We were therefore surprised to note that a small number of animals in the G.gua colony had two ventral openings, suggesting these animals were 

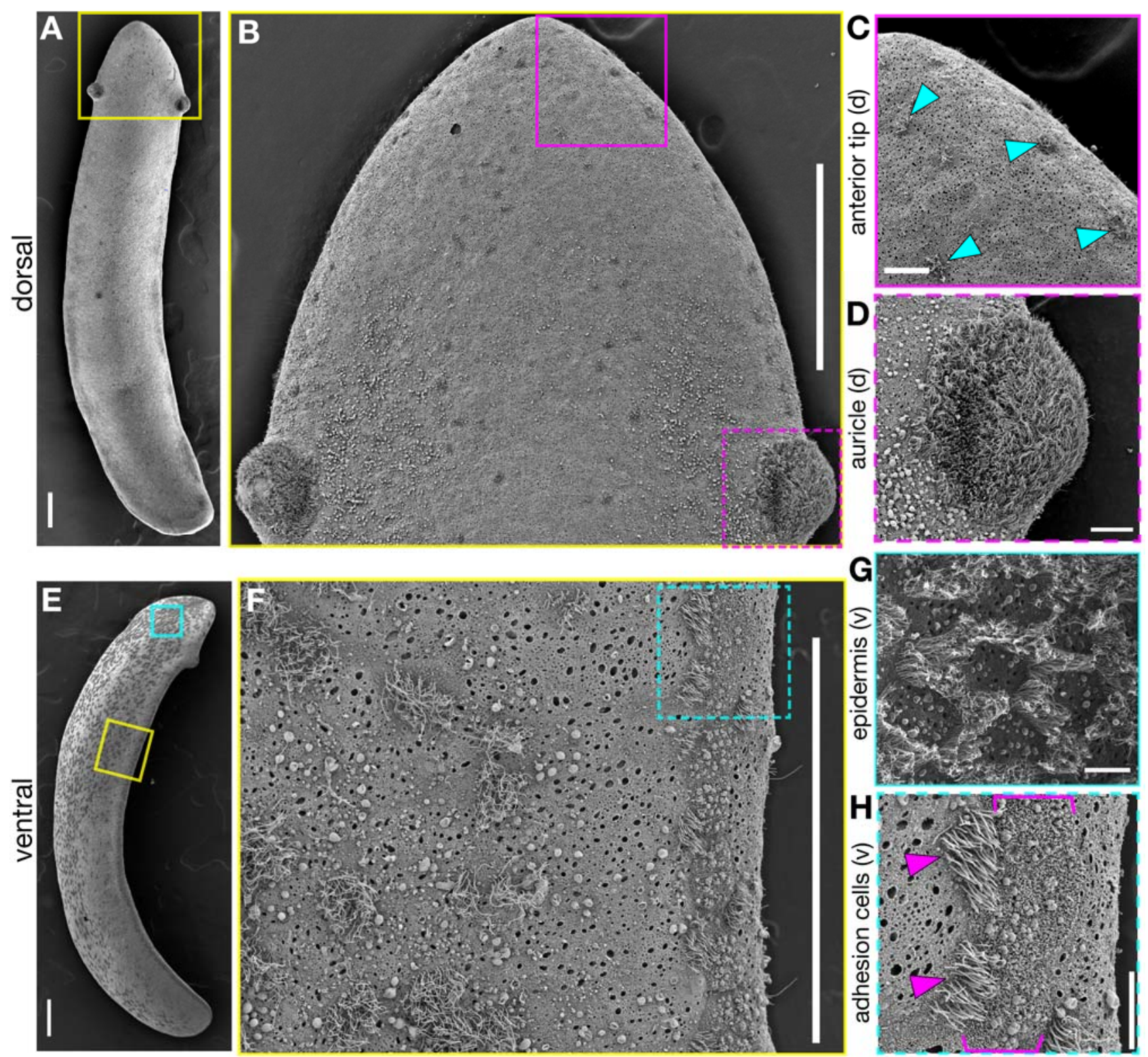

Figure 3. The epidermis of G.gua planarians has a distinct pattern of ciliated cells.

(A) A Scanning Electron Microscopy (SEM) image of a G. gua planarian, viewed from the dorsal side. As seen in (A-D), the dorsal epidermis is largely non-ciliated (sparse individual ciliated cells are indicated by cyan arrowheads) except for the auricles, which are densely ciliated on the dorsal and lateral sides $(B, D)$. The ventral epidermis $(E-H)$ exhibits a distinct pattern of both ciliated cells and non-ciliated cells, which differs from the densely ciliated ventral epidermis seen in many other planarian species. As seen in $(\mathrm{F}, \mathrm{H})$, the decreased density of ciliated cells in the ventral epidermis allows a line of adhesion cells (brackets) to be observed easily by SEM. Individual ventral ciliated cells that lie along the adhesion

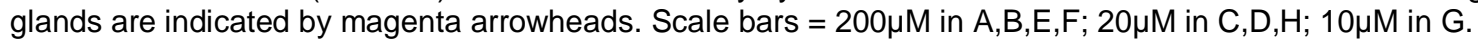

sexualized. We also observed putative testes when examining these rare worms using darkfield microscopy (Figure 4A). We detected these putative sexual animals at an extremely low frequency: $\mathrm{n}=4$ animals in the fall of 2017 and $\mathrm{n}=10$ animals in the fall of 2018 . We closely examined the colony again in the fall of 2019 , but did not detect any animals with two ventral openings. After sequestering these putative sexual animals together for extended periods of time, we never observed any copulatory behavior or eggs, suggesting an immature state of sexualization. To determine if these animals indeed possessed sexual organs, we submitted animals from our 2018 collection for histological analysis. G.gua animals were sectioned sagittally in order to obtain lateral 


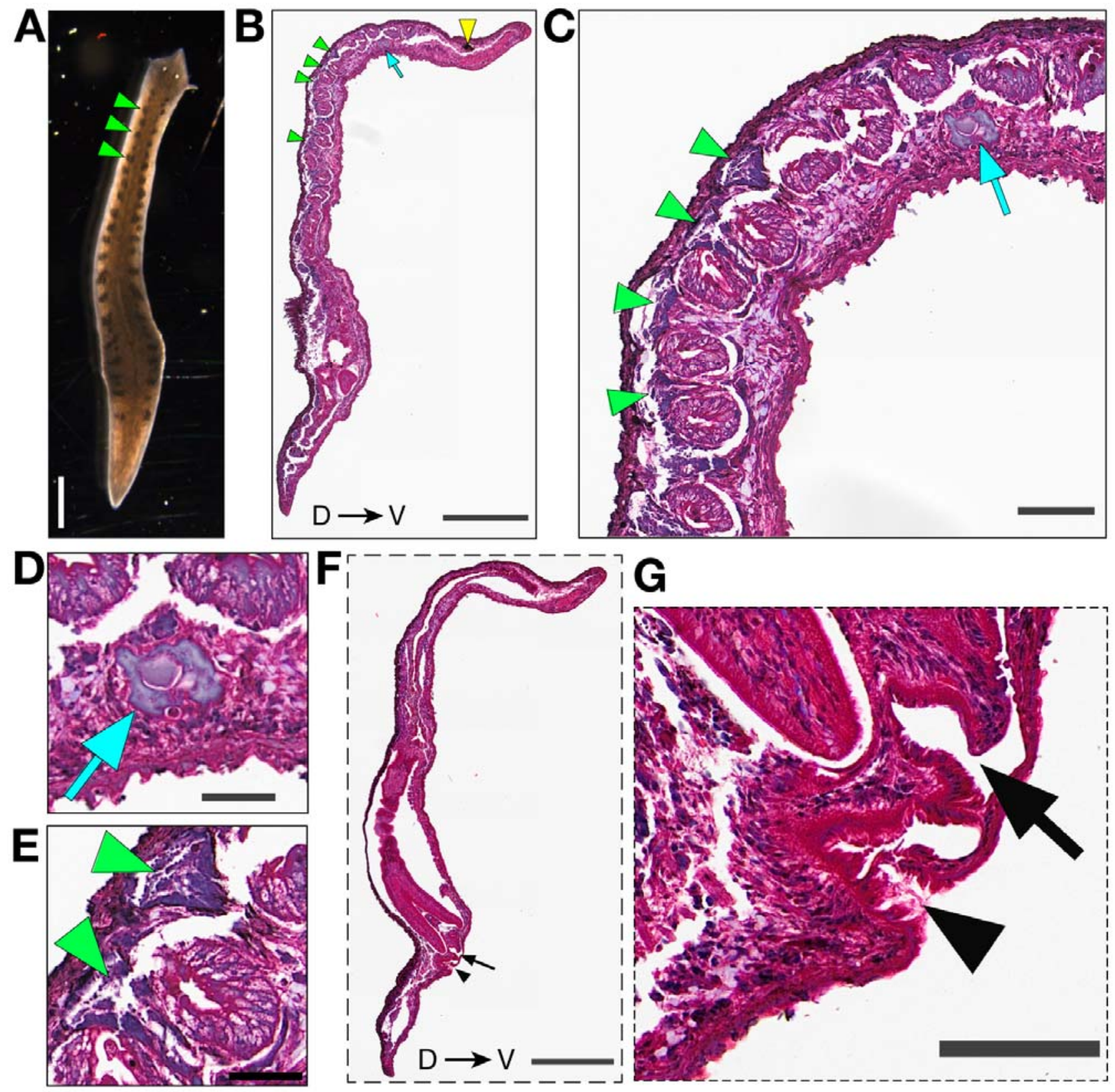

Figure 4. G.gua planarians show sexualized morphology at very low frequency in the lab.

(A) Darkfield image of a rare sexualized animal revealing a clear pattern of dark nodes (green arrowheads) visible dorsolaterally. $(B, F) H+E$ stained histological sections representing two different sagittal planes of an animal with a sexualized appearance. One section (B) reveals an ovary (cyan arrow, B-D) on the ventral side of the worm posterior to the photoreceptors (yellow arrowhead); it also includes testes on the dorsal side (green arrowheads in B,C,E) nested between gut branches. A second more medial sagittal section $(F, G)$ reveals a gonopore (black arrowhead) and atrium (black arrow). Scale bars $=1 \mathrm{~mm}$ in $A, 0.5 \mathrm{~mm}$ in B and F, $100 \mu \mathrm{m}$ in $C$ and $G, 50 \mu \mathrm{m}$ in $\mathrm{D}$ and $\mathrm{E}$.

planes containing both an ovary (Figure 4B-D cyan arrows) and testis (Figure 4B,C,E green arrowheads). As seen in the sexual biotype of S.med, we observed ovaries on the ventral side of G.gua sections in a position posterior to the photoreceptors and just behind the cephalic ganglia (Figure 4B,C, cyan arrow). Sagittal sectioning also revealed details of the medial copulatory region, including a gonopore (Figure 4F,G black arrowhead) and what appears to be an immature copulatory apparatus with an infranucleated epithelial lining and lumen of an atrium (Figure 4F,G 
black arrow and Supplemental Movie S3).These data suggest that G.gua animals are capable of transitioning from a fissiparous (asexual) state to a sexualized state, although it is difficult to conclude without evidence of functional sexual reproduction.

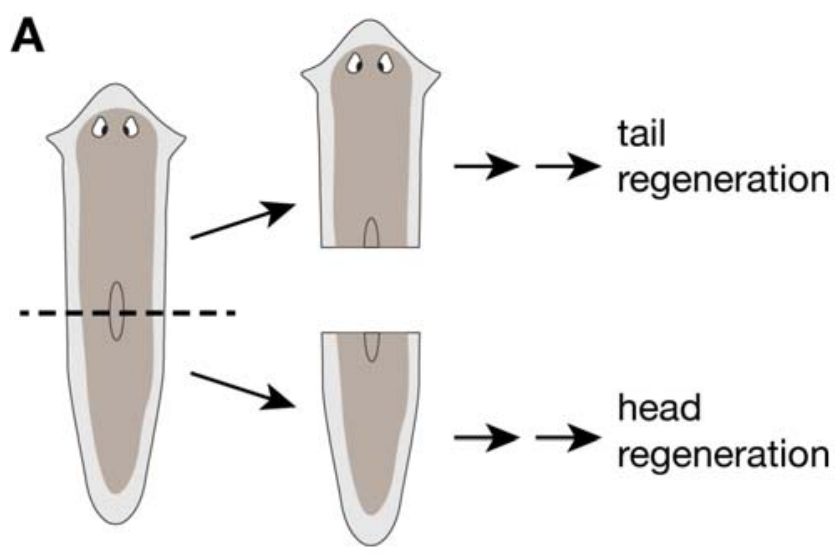

B tail regeneration

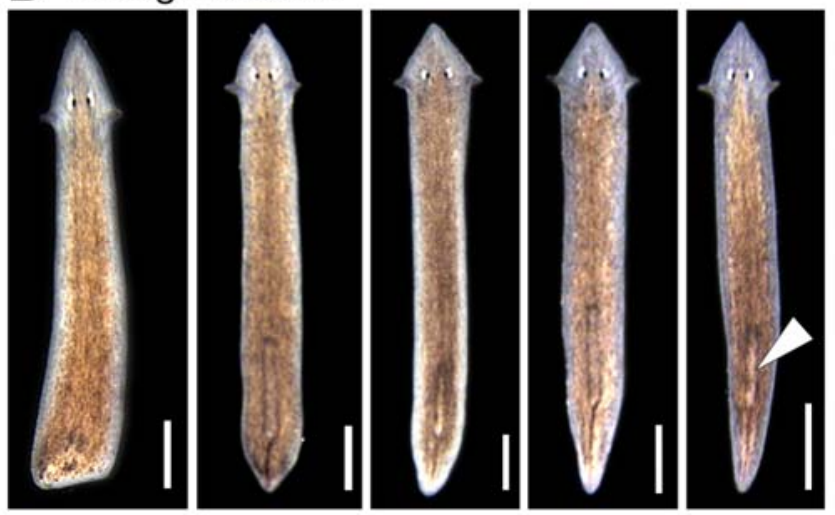

C head regeneration

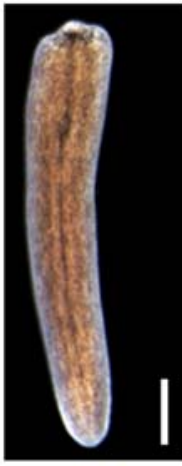

4 dpa

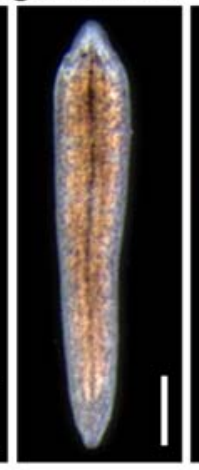

6 dpa

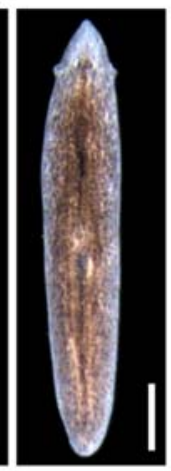

$8 \mathrm{dpa}$

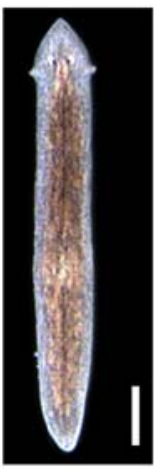

11 dpa 15 dpa
Figure 5. G.gua have high regenerative capacity. (A) Schematic of the amputation strategy used to test the regeneration capacity of newly isolated worms. Amputated G.gua fragments demonstrate a rapid and robust capacity for both new tail regeneration $(B)$ and new head regeneration $(C)$, including the formation of a new pharynx in both fragments (white arrowheads). dpa $=$ days post amputation. Scale bars $=1 \mathrm{~mm}$.

\section{Regenerative Capacity}

Many planarian species have the capacity to regenerate lost tissues, although this ability varies across species. Planarians with limited regenerative abilities often cannot regenerate new heads if amputated near or below the pharynx (22-24). We assayed the regenerative capacity of G.gua animals by bisecting individual animals transversely through the pharynx and monitoring their recovery over the course of 15 days (Figure 5). Both head and tail G.gua fragments demonstrated successful wound healing and survived throughout the course of the experiment. At four days post amputation (dpa), we observed early signs of tissue regeneration in both head (Figure 5B) and tail (Figure 5C) fragments, including the formation of a small, non-pigmented blastema in tail fragments (Figure $5 \mathrm{C}$ ). After six days, tail fragments showed signs of newly regenerated photoreceptors (e.g., two spots of dark pigment at the anterior wound site) and after 8 days the eyespots were clearly and completely formed (Figure 5C). All worm fragments had fully regenerated within fifteen days. These data clearly show that G.gua planarians have a robust regenerative capacity.

\section{Stem cell population}

The regenerative capacity of planarians depends on a population of adult stem cells, also known as neoblasts, that bear many unique properties (25). Not only do these stem cells self-renew and differentiate as needed during both regeneration and normal homeostasis, but they do so indefinitely (5). After establishing the high regenerative capacity of G.gua, we sought to examine the number, distribution, and functional properties of presumptive G.gua stem cells.

Figure 6. G.gua planarians have a higher tolerance for 

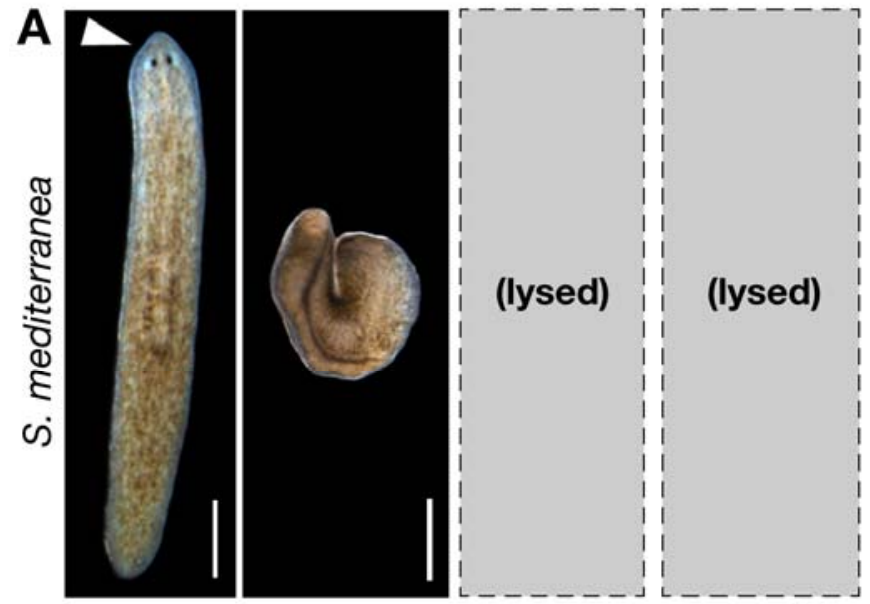

16 dpi

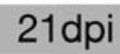

28 dpi

35 dpi
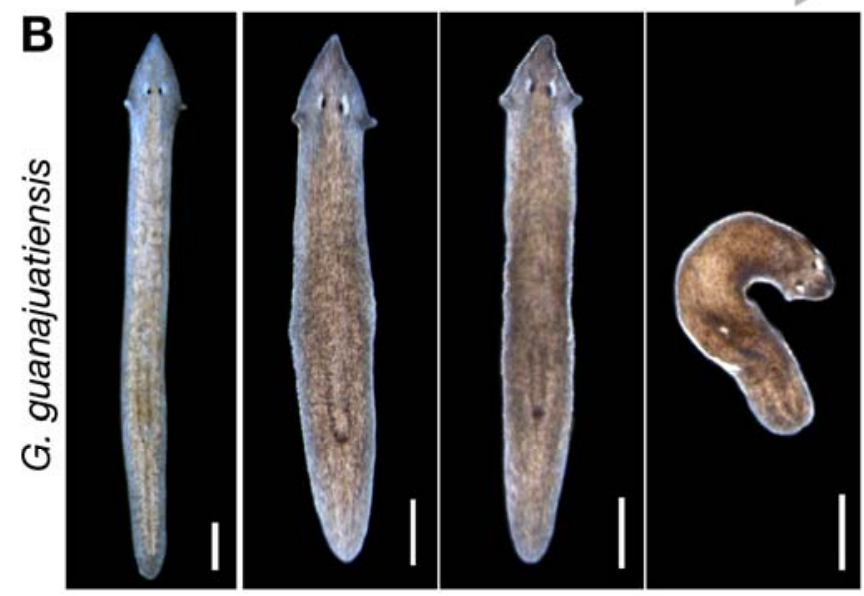

C

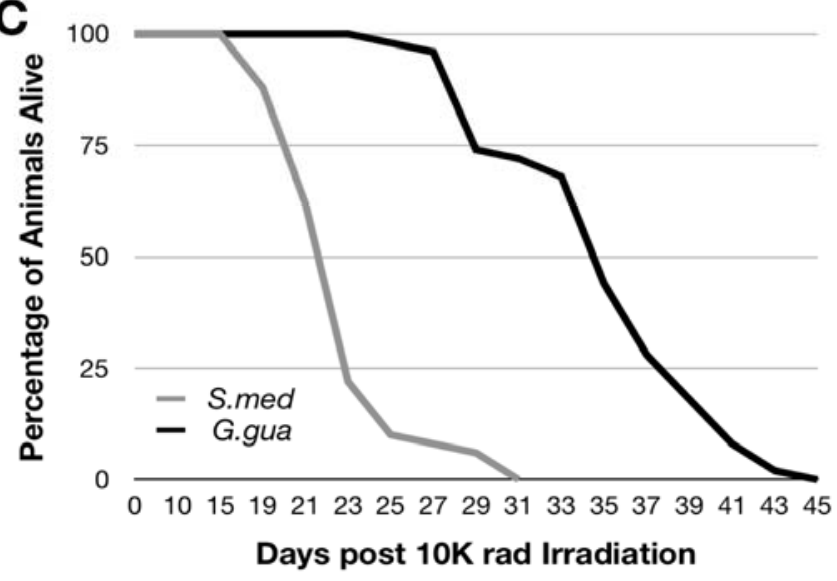

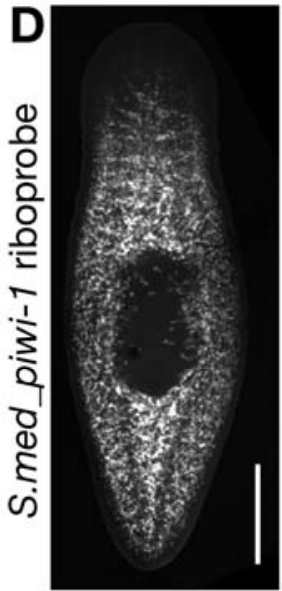
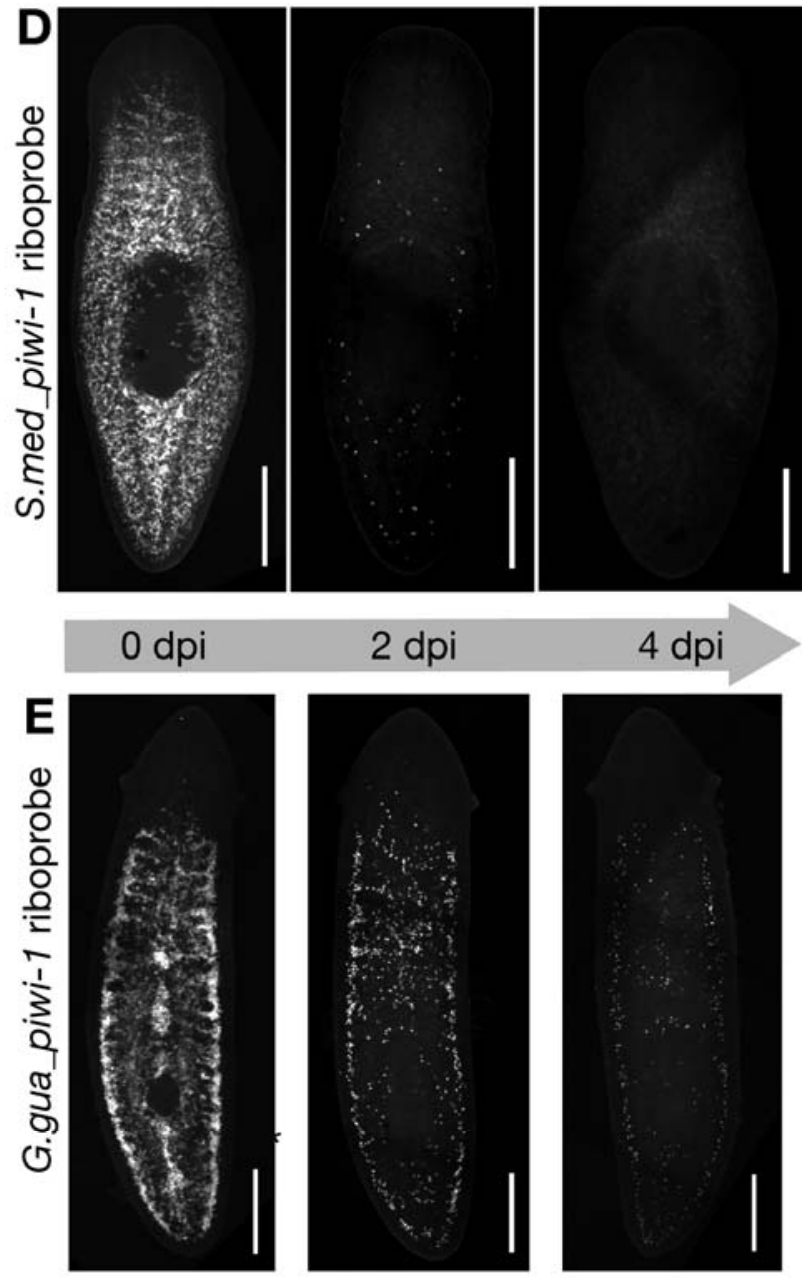

\section{2 dpi}
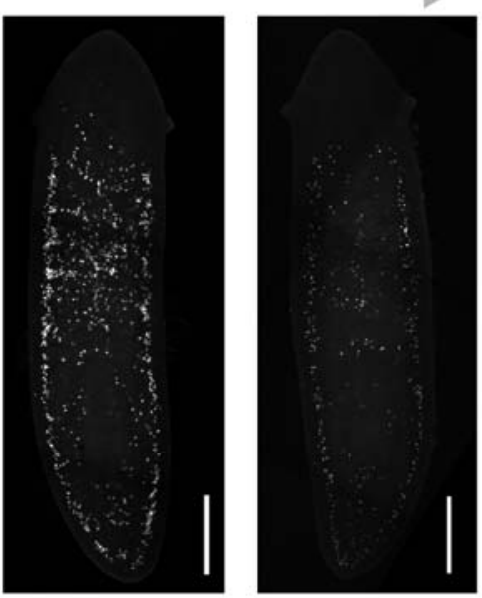

F

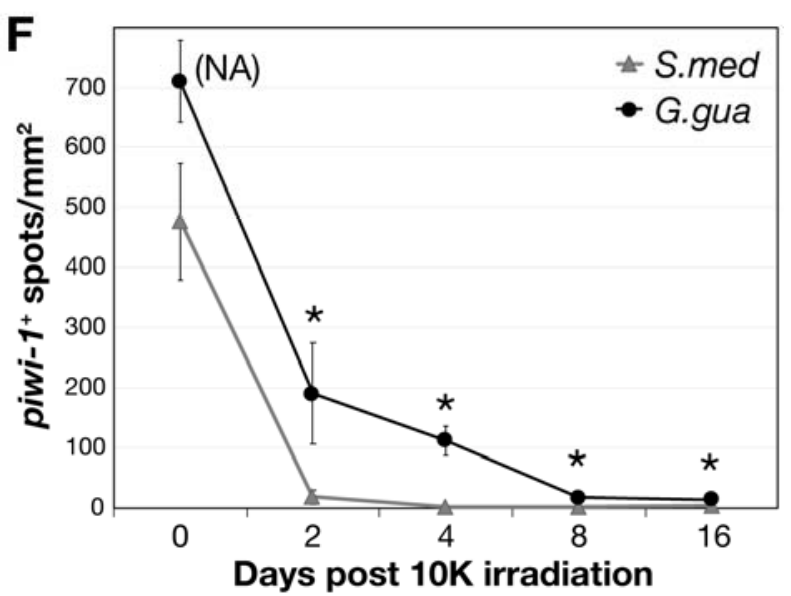

\section{$\square$-radiation than S.med planarians.}

50 worms per S.med (A) and G.gua (B) species were exposed to the same dose of $\square$-radiation (10K rads) and monitored over time. Images are representative of the phenotype seen in most worms at a given time point (days post-irradiation; dpi). (C) Survival data from the experiment represented in A and B was recorded at the indicated days post radiation treatment and plotted as shown. (D) in situ hybridization images of representative S.med worms or (E) G.gua worms fixed at the indicated time points post $\square$-radiation and stained with species-specific piwi-1 riboprobes. (F) Quantitation of piwi- $1^{+}$cells for the experiment shown in $\mathrm{D}$ and $\mathrm{E} ; \mathrm{n}=4-5$ worms at each time point. $\mathrm{NA}=$ density of piwi-1 signal is too high at the indicated time point for accurate quantitation. ${ }^{*}=p<0.02$. All scale bars $=500 \mu \mathrm{M}$. 
To assess the functional conservation of G.gua stem cells, we turned to a well established assay of planarian stem cell function. In other highly regenerative planarian species, including $S . m e d$, it is known that neoblasts respond to ionizing radiation in a reproducible and dosedependent manner $(26,27)$. Doses $\geq 6000(6 \mathrm{~K})$ rads of $\square$-radiation cause rapid depletion of $S$. med stem cells, which then leads to homeostasis failure and death. To address whether G.gua stem cells respond similarly to $\square$-radiation, we exposed both G.gua and S.med animals to $10 \mathrm{~K}$ rads of $\square$-radiation and measured its effects (Figure 6). Notably, most $S$. med animals lysed as expected within 23 days days post irradiation (dpi; Figure 6A,C). However, irradiated G.gua maintained normal morphology for nearly $28 \mathrm{dpi}$ and the majority did not lyse until $35 \mathrm{dpi}$ (Figure 6B,C). Importantly, these results were repeated in multiple independent experiments and consistently showed the same species-specific effect.

After observing such a significant phenotypic difference between G.gua and S.med in response to lethal $\square$-radiation, we sought to determine the direct effects of this treatment on stem cell number and distribution within G.gua animals. To accomplish this, we created a molecular marker of G.gua stem cells by cloning the G.gua homolog of piwi-1, a gene that is highly expressed and enriched in the stem cells of other planarian species $(28,29)$. We generated a fluorescent riboprobe to this sequence and performed in situ hybridization in fixed G.gua worms. As seen in Figure 6E (0dpi), G.gua_piwi-1 probe not only detects an abundant population of putative stem cells, but the distribution of these cells is very similar to that of S.med piwi $1^{+}$cells (Figure 6D, 0dpi), including their absence from the anterior tip of the head and the pharynx. To analyze the effect of lethal $\square$-radiation on G.gua_piwi $1^{+}$stem cells, we fixed animals at multiple dpi and performed in situ hybridization with species-specific piwi-1 riboprobes (Figure 6E). Intriguingly, lethally irradiated G.gua animals maintained a significant number of G.gua_piwi ${ }^{+}$stem cells for at least 8 days after lethal $\square$-radiation (Figure 6E, F), whereas S.med_piwi $1^{+}$stem cells were rapidly depleted within 2 days as expected (Figure 6D, F). Although this finding did correlate with the relative difference in survival of G.gua animals compared to S.med (Figure 6A-C), we were still surprised that a small but significant number of G.gua_piwi $1^{+}$stem cells persisted for so long after such a high dose of $\square$-radiation.

\section{Molecular Characterization}

Given that the morphological and functional analyses of G.gua animals revealed several interesting and distinct features, we characterized them further. Our first step was to determine their ploidy using a recently optimized protocol for chromosome preparation and karyotyping in planarians and other organisms (30). After treating G.gua tissue with colchicine to block any cycling cells in metaphase, we then swelled, fixed, squashed, and stained it (using DNA dye) to visualize the metaphase chromosomes. Optimal spreads revealed individual cells with clear boundaries and distinct, non-overlapping chromosomes. From these spreads, we identified four diploid chromosome pairs per G.gua metaphase cell (Figure 7A).

We then generated a G.gua transcriptome to: 1) validate the Girardia genus classification with molecular data; and 2) generate a useful resource for future studies. To create this dataset, we isolated total RNA from a single G.gua animal and reverse transcribed an mRNA library using polyA selection. After sequencing and assembling reads from this library, we analyzed the resulting transcriptome for completeness using BUSCO analysis, or "Bench-marking Single Copy Orthologs" (32); (Figure 7B). The assembled G.gua transcriptome is largely complete, as approximately $90 \%$ of the 978 metazoan BUSCOs matched completely to assembled G.gua transcripts. We then 
A G. guanajuatiensis karyotype

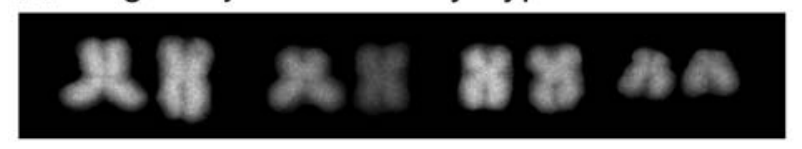

B

nonredundant

peptides

peptides

transcripts

L

Missing (M)

Fragmented (F) $\square$ Complete (C) and Single-copy (S)
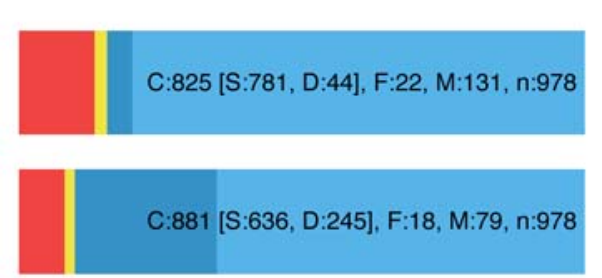

$C: 876$ [S:592, D:284], F:17, M:85, n:978

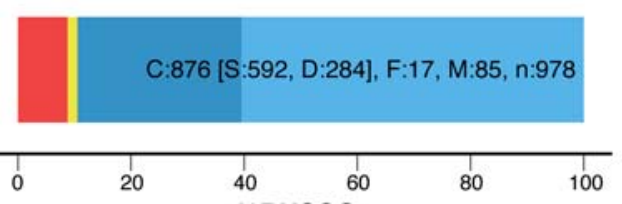

$\%$ BUSCOs
D phylogenetic tree (transcriptome-based)

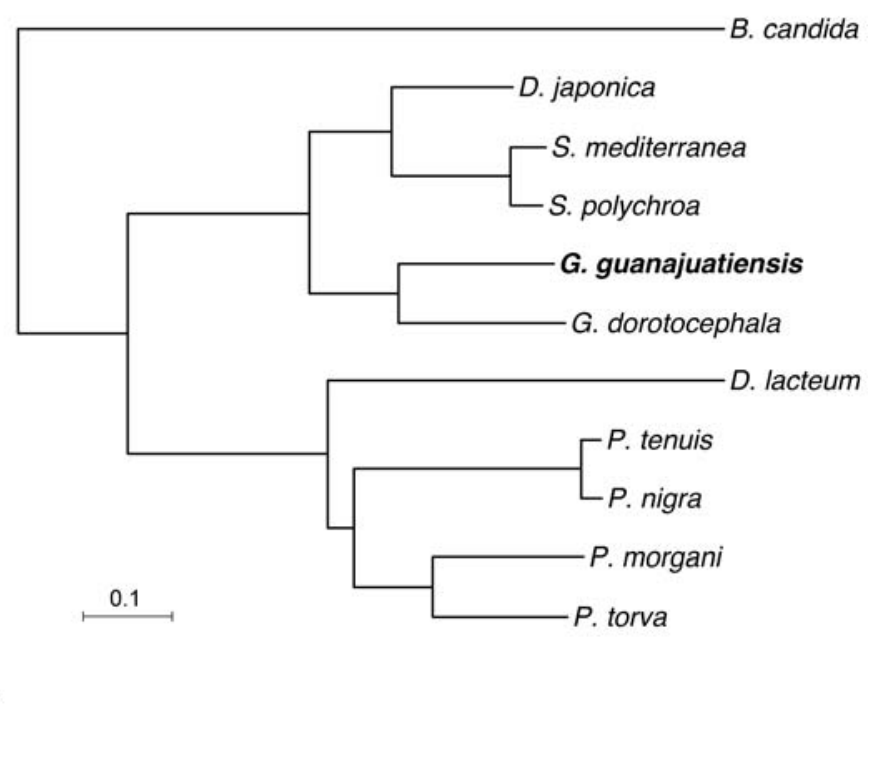

C

assembled transcripts

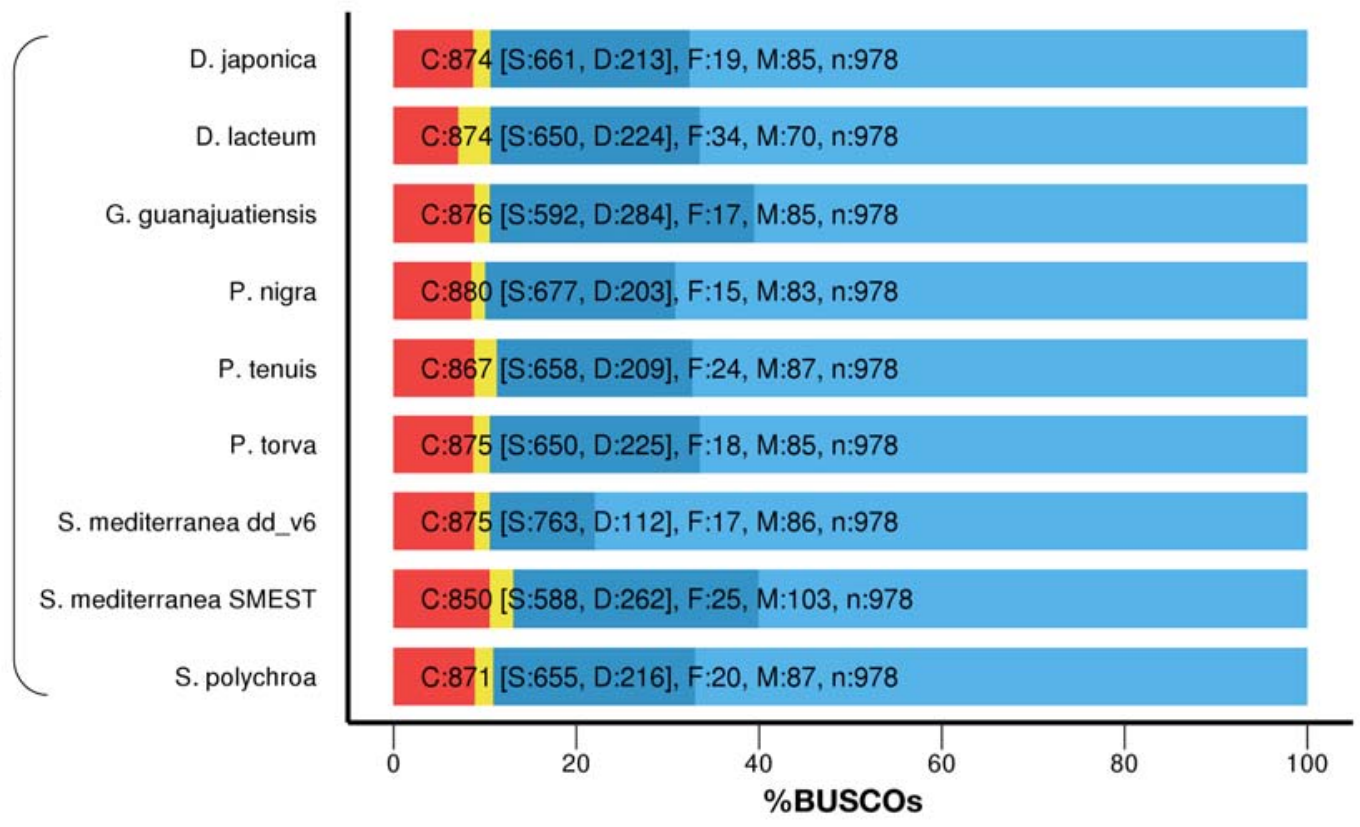

Figure 7. G.gua are diploid planarians in the Girardia genus.

(A) Karyotype of cells in G.gua tail tissue. (B) Transcriptome completeness evaluation of the newly assembled G.gua transcriptome using 978 metazoan BUSCOs. (C) Comparison of the newly assembled G.gua transcriptome to 8 other assembled transcriptome downloaded from the PlanMine database (31). (D) Phylogenetic tree created using 3,163 concatenated single-copy putative orthologs from 11 triclad flatworm species. Scale bar unit = average number of substitutions per site.

asked how this newly assembled transcriptome compared with those of other planarian species. We therefore downloaded eight additional transcriptomes from the PlanMine database (31) and analysed each set of assembled transcripts using the same BUSCO parameters as used for our G.gua transcriptome analysis. As shown in Figure 7C, the G.gua transcriptome is comparable to that of other planarian transcriptomes, including those that have been experimentally validated in many publications $(31,33,34)$, in terms of completeness. 
After generating this new G.gua transcriptome, we compared sequences of specific gene orthologs with those of multiple flatworm species and created a phylogenetic tree that includes the guanajuatiesis species (Figure 7C). This analysis supports our classification of these newly documented planarians as Girardia, as they clearly cluster with Girardia dorotocephala when analyzed with 10 other flatworm species representing 6 different genera.

\section{DISCUSSION}

Planarians are best known for their incredible regenerative abilities and negligible senescence, both of which depend on the maintenance of a dynamic and heterogeneous population of adult stem cells. Although some previous studies have identified species-specific differences that underlie regeneration competence in planarians (22-24), a lack of robust genomic resources for more than one species impedes the planarian field from using comparative genomic approaches. This lack is due, in large part, to the enduring difficulty of assembling planarian genomes. To advance our understanding of the cellular and molecular basis of planarian regeneration, and other fundamental mechanisms shared across the animal kingdom, we need to overcome this obstacle. Although the assembly of planarian genomes has proven difficult in the past (34), recent advances in planarian genome assembly (35) and bioinformatic tools that address genome heterozygosity (36) suggest that this problem is indeed tractable and will pave the way for future genome assembly and comparison projects in multiple planarian species.

Here we characterize a previously undescribed planarian species, Girardia guanajuatiensis. This new species has a diploid genome and is putatively asexual, although we cannot fully exclude the possibility that G.gua can be sexualized. Notably, whereas the asexual biotype of the wellstudied S.med species can be distinguished from the sexual biotype by its karyotype (evidence of a large chromosomal translocation is only present in asexual S.med), we do not observe any obvious chromosomal anomalies in the G.gua karyotype (Figure 7A). Nevertheless, under laboratory conditions, most G.gua animals appear to maintain an asexual and largely diploid status. Sexualization of Girardia species has been documented in the wild and in the laboratory, which fits with our observation of rare sexual G.gua. Both G. tigrina and G. dorotocephala alternate their reproductive status seasonally in nature, becoming sexual in cooler months and asexual in warmer months (37). Additionally, both species can be induced to sexualize in the laboratory upon being fed ground up tissue from the exclusively sexual Polycelis nigra $(38,39)$. In these examples, sexualization was induced in response to a clear and definable change in temperature or in food, whereas our G. gua animals were maintained in a temperature controlled environment and fed a consistent diet of calf liver (see Materials and Methods). Spontaneous sexualization of $G$. tigrina laboratory animals has been reported on multiple occasions $(17,40)$ and examples of these events share some similarities with our observation in G. gua, such as an immature copulatory apparatus and sterility. Nevertheless, these reports describe some features that we did not readily observe in our limited number of putatively sexual G.gua, such as hyperplastic ovaries and the presence of supernumerary copulatory apparatuses.

Our examination of G.gua also revealed two unique, likely interrelated, features: an unusual mode of locomotion and a distinctive pattern of ciliated cells on their outer epithelium. Planarian locomotion has long been known to be propelled by the concerted beating of motile cilia that nearly completely cover the outer epithelium $(11,14)$. These cilia beat against a layer of secreted mucus, which allows them to achieve a smooth gliding motion. However, G.gua appear to have two modes of locomotion: the typical smooth gliding seen in most planarian species and a peristaltic-like 
motion that is more similar to the "inch-worming" phenotype observed upon chemical or genetic perturbation of motile cilia $(18,41)$. As this phenotype is a well characterized cilia deficiency, we immediately examined the outer epithelium of G.gua. Indeed, we found that its appearance is dramatically different from that of other well-studied planarians. The patchy pattern of cilia on the ventral surface of G.gua likely explains its frequent peristaltic-like motion. As this pattern is typically only observed upon cilia protein depletion (i.e., by RNAi), it suggests that G.gua may regulate cilia gene expression, cilia protein production, and/or the differentiation of mature epithelial cells differently than other planarian species. Given the morphological and functional similarity of this outer mucociliary epithelium to that of many human structures, including the lungs and oviducts, G.gua offer an opportunity to identify and study the cellular mechanisms that are dysregulated in various human pathologies affecting these epithelia.

Another way in which G.gua distinguish themselves from S.med is in their response to high doses of ionizing radiation. We were surprised to observe the significantly long survival time of G.gua animals post $10 \mathrm{~K}$ rads of $\square$-radiation, a dose that is approximately 50 times higher than a standard clinical dose used to treat human cancers. We were even more surprised to observe the persistence of piwi- $1^{+}$stem cells for such an extended period of time after radiation treatment, particularly as compared to those of S.med. Whereas G.gua animals exposed to $10 \mathrm{~K}$ rads of $\square$ radiation lived approximately $1.5 x$ longer than $S$. med animals treated with this dose, a significant number of G.gua piwi-1 $1^{+}$stem cells persisted at least $4 x$ longer than S.med piwi-1 ${ }^{+}$stem cells. There are many possible explanations for this differential response, including differences in checkpoint mechanisms leading to cell cycle arrest and/or differences in stem cell number. As the cellular response to genotoxic stress has critical implications in human cancer progression and metastasis, studies that dissect the molecular basis of radiation tolerance in G.gua will likely provide important insight into the molecular mechanisms underlying cancer therapy resistance.

The collection and characterization of this previously undescribed planarian species, Girardia guanajuatiensis, is a valuable addition to the study and documentation of animals in the Turbellaria class. Its unique characteristics raise many interesting questions about their evolution and development, which will undoubtedly spark many future studies.

\section{CONCLUSIONS}

Here we describe a newly documented species of planarian flatworm, Girardia guanajuatiensis. We show that it is a highly regenerative, putatively asexual, diploid species that bears morphological and genetic characteristics of the genus Girardia. We also document two distinct features of this species that distinguish it from most other documented species. By expanding the number and depth of planarian species characterizations, our study will facilitate both comparative studies and those that specifically address the cellular and molecular basis of the unique features of G.gua.

\section{METHODS}

\section{Collection and Husbandry}

Animals were collected from a site near the Presa San Revato in the Parque Bicentenario in Guanajuato City, Mexico between July 31st and August 2nd of 2016. Animals were transported back to the laboratory and housed in disposable plastic food storage containers filled with Montjuic water (13). The containers were stored in a dark cabinet at room temperature (22-25C). Worms used in experiments were starved $\geq 7$ days prior to selection for experiments. 


\section{Imaging}

All images were adjusted for brightness and contrast using Fiji (42). High speed imaging of auricle cilia was captured using an ORCA-Flash4.0 V2 C11440-22CU camera from Hamamatsu mounted on a Zeiss Axiovert 200 microscope, as previously described (12).

\section{Light Microscopy and SEM}

Light microscopy images of live worm morphology were captured with both Leica M205 and M165 stereomicroscopes. Scanning Electron Microscopy samples were processed as in (43). SEM imaging was performed on a Zeiss Merlin. SEM images were processed with the CLAHE plugin (settings: 127, 256, 1.5; available at:

https://github.com/axtimwalde/mpicbg/tree/master/mpicbg/src/main/java/mpicbg/ij/clahe).

\section{Histology}

Tissue processing, section preparation, H\&E and special staining were performed as previously conducted in the core lab with modifications $(44,45)$. Briefly, for tissue processing and section, planarian worms were fixed with 4\% PFA in PBS (diluted from 16\% (wt/vol) aqueous solution, Electron Microscopy Sciences, cat\# 15710) for $24 \mathrm{hr}$ at $4^{\circ} \mathrm{C}$, then rinsed well with $1 \times \mathrm{PBS}$, dehydrated through graded ethanol $(30 \%, 50 \%, 70 \%)$ and processed with a PATHOS Delta hybrid tissue processor (Milestone Medical Technologies, Inc, MI). Using a Leica RM2255 microtome (Leica Biosystems Inc. Buffalo Grove, IL), sections were cut in $5 \mu \mathrm{m}$ thickness and mounted on Superfrost positive charged microscope slides (cat\# 12-550-15, Thermo Fisher Scientific). H\&E routine stain was performed using Leica ST5010 Autostainer XL and Leica ST Infinity H\&E Staining System kit (VWR\#3801698) according to the manufacturer's instructions. Resultant slides were imaged on an Olympus America Slide Scanner.

\section{In-situ Hybridization}

In-situ hybridization was performed as previously described using Digoxigenin labeled riboprobes and tyramide amplification of their signal $(46,47)$. After probe hybridization, signal development, and mounting on slides, animals were imaged on a CSU-W1 with a Nikon base and Prior plate loading robot as described (48). Fully automated image acquisition was obtained by first imaging whole slides using a 4 X $0.2 N A$ objective. Individual worms were segmented and z-stacks acquired using a 10X $0.45 \mathrm{NA}$ objective. Stitching and spot quantification were performed in Fiji using custom macros (https://github.com/jouyun/smc-macros).

\section{Irradiation}

Irradiation was done with a Gammacell 40 Exactor (MDS Nordion) at a dose rate of $85 \mathrm{Rad} / \mathrm{minute}$.

\section{Karyotyping}

Karyotyping was carried out as previously described (30).

\section{RNA Extraction and Sequencing}

Total RNA from one worm was extracted with TRIzol ${ }^{\mathrm{TM}}$ (Invitrogen) using the standard protocol. An mRNAseq library was generated from 100ng of high quality total RNA, as assessed using the Bioanalyzer (Agilent). The library was made according to the manufacturer's directions for the TruSeq Stranded mRNA LT Sample Prep Kit - set B (Illumina, Cat. No. RS-122-2102). The 
resulting short fragment library was checked for quality and quantity using the LabChip GX (PerkinElmer) and Qubit Fluorometer (Life Technologies). The library was then sequenced as 100bp paired reads on two rapid-run flow cells using the Illumina HiSeq 2500 instrument. Following sequencing, Illumina Primary Analysis version RTA 1.18.64 and bcl2fastq2 v2.18 were run to demultiplex the sequencing reads and generate FASTQ files.

\section{Transcriptome Assembly}

Transcriptome assembly was created with Trinity (version: v2.2.0; parameters: --trimmomatic -min_contig_length 300) (49). Post-assembly vector trimming and contaminate filtering was performed with SeqClean (version: 20110222; parameters: - - 300) (50). Transcriptome assembly consists of 183,422 sequences with a mean GC content of $32 \%$.

Sequences were translated with Transdecoder (version: v2.0.1) (51) and collapsed into longest isoforms with cd-hit (version: 4.6, parameters: -c .95 -G 0 -aS .5) (52). The resulting non-redundant file of predicted proteins consisted of 23,850 proteins of which 10,303 contain both start codons and stop codons; 5,993 contain stop codons, but not start codon; 2,409 contain a start codon, but not a stop codon; and 5,145 contain neither stop, not start codons.

\section{Transcriptome Completeness Assessment}

BUSCO (version: 3.1 .0 ; parameters: -I metazoa_odb9) (32) was used for completeness analysis. BUSCO found $89.5 \%$ of metazoan single-copy orthologs in the G.gua transcriptome assembly with only $8.8 \%$ of single-copy orthologs missing. (short summary:

$\mathrm{C}: 89.5 \%[\mathrm{~S}: 60.5 \%, \mathrm{D}: 29.0 \%], \mathrm{F}: 1.7 \%, \mathrm{M}: 8.8 \%, \mathrm{n}: 978)$. BUSCO analyses of other planarian transcriptomes were performed on transcriptomes downloaded from Planmine.

\section{Phylogenetic Analysis}

Preliminary BLAST (53) analysis suggested Girardia guanajuatiensis as most closely related to previously published Girardia species. To refine the position of this species in the planarian phylogenetic tree OrthoMCL (version: v2.0.9) (54) was used to define putative ortholog groups from G. guanajuatiensis and 11 published transcriptomes: Dendrocoelum lacteum, Polycelis nigra, Polycelis tenuis, Schmidtea mediterreanea, Planaria torva (31), Dugesia japonica (GFJY01000000.1), Schmidtea polychroa (GFKA00000000.1), Girardia dorotocephala (GEIG01000000.1), Phagocata morgani (GEKK01000000.1), Phagocata gracilis (GEGP01000000.1) and Bdelloura candida (GFKB01000000.1).

Gene groups with no more than one sequence from each species and present in at least ten of the selected species were separately aligned with MUSCLE (version: v3.8.31) (55), trimmed with trimAl(version: v1.4.rev22 ; parameters: -automated1) (56) and then concatenated and cleaned with phyutility (version: v.2.2.6; parameters -clean .5) (57). Phylogenetic tree was created using RAxML-NG (version: 0.8.1 BETA; parameters: --model PROTGTR+G --seed 123) (58).

\section{List of abbreviations}

G.gua = Girardia guanajuatiensis

S. med = Schmidtea mediterranea

Dpa = days post amputation

Dpi $=$ days post irradiation 


\section{DECLARATIONS}

\section{Ethics approval and consent to participate}

Not applicable.

\section{Consent for publication}

Not applicable.

\section{Availability of data and materials}

Original data underlying this manuscript can be accessed from the Stowers Original Data

Repository at http://www.stowers.org/research/publications/libpb-1535. The Girardia

guanajuatiensis transcriptome is deposited with the NCBI Bioproject: PRJNA551297. NCBI

assigned G.gua with taxonomy ID: 2592332 and temporary name Girardia sp. N. ER-2019.

\section{Competing interests}

The authors declare that they have no competing interests.

\section{Funding}

ASA is funded from NIH Grant R37GM057260-20, the Stowers Institute for Medical Research and the Howard Hughes Medical Institute. EMD is funded from a Pilot Project award under NIH Grant GM121327 and The University of Kentucky. The 2016 CdeC Workshop for Developmental Biology was funded by a grant from the Center for Teaching, Learning, \& Outreach (CTLO).

\section{Authors' contributions}

ASA and CdeC located and collected the specimens suspecting them to be a previously undescribed species. EMD, SHN, CGH, and EJR contributed to experimental design. CGH completed histological specimen preparations, and RNA isolation. JD completed the regeneration experiment and live imaging of regenerating fragments. CGH and EMD completed live worm imaging, irradiation, and stem cell staining experiments. SM performed all fluorescent imaging and image analyses. SHN performed all SEM and pharynx imaging. LG and CGH performed all chromosome preparations and karyotyping. EJR completed phylogeny and transcriptome analyses. EMD and SHN wrote the manuscript.

\section{Acknowledgements}

We thank all members of the Sánchez Alvarado Lab for discussion and insightful comments. We thank the Histology Core and the Light and Electron Microscopy Core at Stowers Institute for Medical Research for SEM sample preparation, histological preparation, and slide scanner imaging. We particularly thank Yongfu Wang for assistance with histology and Jeff Lange for his assistance in capturing the live, high speed images of beating cilia. We also acknowledge the assistance of Sofia Robb at the Stowers Institute for Medical Research for help with OrthoMCL, as well as Brian Fleharty and the Stowers Institute for Medical Research Molecular Biology Core facility for library preparation and sequencing.

In 2016, the Clubes de Ciencia México Workshop for Developmental Biology included: Leslie Yareli Govea-Martínez, Cristell Hernández-Fonseca, Benjamín Pérez-Sánchez, Israel Torres-Alba, Maria Ramirez-Morales, Erick Navarro-Delgado, Nohemí Carolina García-Rodríguez, Arizbeth Plascencia, José Luis Rodríguez-Ortiz, L. Gerardo Moreno-Ciénega, Adrian Maciel-Avalos, Porfirio 
Gallegos-Casillas, Mónica Vázquez Guerrero, Eduardo Zamudio de la Cruz, Marcos RomeroPartida, Rodrigo Jonathan Martinez-Espinosa, Abigail Zuñiga-Arenas, Sofía Quiroz Yebra, Sara Elena Ambriz-Piña, José Segoviano, Karla Fernández (undergraduate students); Fernando Longoria Vázquez (course assistant); Heather Leigh Curtis and David Angeles-Abores (course organizers).

\section{Additional Files}

- $\quad$ supplemental movie S1 (movie, .mp4); wild-type G.gua worm motility

- $\quad$ supplemental movie S2 (movie, .mp4); motile cilia on auricle of wild-type G.gua worm

- supplemental movie S3 (movie, .mp4); serial sections of copulatory region

- additional file 1 (BUSCOs) (pdf, .pdf); BUSCO Results for all transcriptomes in Figure 7C

\section{REFERENCES}

1. Elliott SA, Sánchez Alvarado A. The history and enduring contributions of planarians to the study of animal regeneration. Wiley Interdiscip Rev Dev Biol. 2013 May;2(3):301-26.

2. Kenk R, Oceanography and Limnology Program (Smithsonian Institution), United States. Freshwater planarians (Turbellaria) of North America [Internet]. 1972. Available from: http://dx.doi.org/10.5962/bhl.title.4020

3. Yang H-M, Sluys R, Kawakatsu M, Min G-S. New molecular sequences for two genera of marine planarians facilitate determination of their position in the phylogenetic tree, with new records for two species (Platyhelminthes, Tricladida, Maricola). Zookeys. 2018 Aug 9;(781):117.

4. Jones HD. A new species of land planarian (Platyhelminthes: Tricladida: Terricola:

Rhynchodemidae) from South Africa, with a list of African terrestrial planarian species [Internet]. Vol. 39, African Zoology. 2004. p. 31-40. Available from: http://dx.doi.org/10.1080/15627020.2004.11407283

5. Newmark PA, Sánchez Alvarado A. Not your father's planarian: a classic model enters the era of functional genomics. Nat Rev Genet. 2002 Mar;3(3):210-9.

6. Vila-Farré M, Rink JC. The Ecology of Freshwater Planarians [Internet]. Methods in Molecular Biology. 2018. p. 173-205. Available from: http://dx.doi.org/10.1007/978-1-4939-7802-1_3

7. Brøndsted HV. Planarian Regeneration. Oxford, New York: Pergamon Press; 1969. 276 p. (International Series of Monographs in Pure and Applied Biology).

8. Villar D, Li MH, Schaeffer DJ. Toxicity of organophosphorus pesticides to Dugesia dorotocephala. Bull Environ Contam Toxicol. 1993 Jul;51(1):80-7.

9. Hagstrom D, Cochet-Escartin O, Collins E-MS. Planarian brain regeneration as a model system for developmental neurotoxicology. Regeneration (Oxf). 2016 Apr;3(2):65-77.

10. Ding X, Song L, Han Y, Wang Y, Tang X, Cui G, et al. Effects of Fe3+ on Acute Toxicity and Regeneration of Planarian (Dugesia japonica) at Different Temperatures. Biomed Res Int. 2019 Aug 22;2019:8591631.

11. Rompolas $P$, Azimzadeh J, Marshall WF, King SM. Analysis of ciliary assembly and function in planaria. Methods Enzymol. 2013;525:245-64. 
12. Thi-Kim Vu H, Rink JC, McKinney SA, McClain M, Lakshmanaperumal N, Alexander R, et al. Stem cells and fluid flow drive cyst formation in an invertebrate excretory organ. Elife [Internet]. 2015 Jun 9;4. Available from: http://dx.doi.org/10.7554/eLife.07405

13. Accorsi A, Williams MM, Ross EJ, Robb SMC, Elliott SA, Tu KC, et al. Hands-On Classroom Activities for Exploring Regeneration and Stem Cell Biology with Planarians. Am Biol Teach. $2017 \operatorname{Mar} 1 ; 79(3): 208-23$.

14. Smales LR, Blankespoor HD. The epidermis and sensory organs of Dugesia tigrina (Turbellaria: Tricladida). Cell Tissue Res. 1978 Oct 1;193(1):35-40.

15. Kenk R. Freshwater triclads (Turbellaria) of North America. IV. The polypharyngeal species of Phagocata. Smithson Contrib Zool [Internet]. 1970; Available from: https://repository.si.edu/bitstream/handle/10088/5341/SCtZ-0080-Lo_res.pdf?sequence=2

16. Sluys R, Littlewood DTJ, Bray RA. Towards a phylogenetic classification and characterization of dugesiid genera (Platyhelminthes, Tricladida, Dugesiidae): A morphological perspective. Taylor and Francis; 2001.

17. Sluys R, Kawakatsu M, Ponce de León R. Morphological stasis in an old and widespread group of species: Contribution to the taxonomy and biogeography of the genus Girardia (Platyhelminthes, Tricladida, Paludicola). Stud Neotrop Fauna Environ. 2005 Aug 1;40(2):15580.

18. Rompolas P, Patel-King RS, King SM. An outer arm Dynein conformational switch is required for metachronal synchrony of motile cilia in planaria. Mol Biol Cell. 2010 Nov 1;21(21):3669_ 79.

19. Rink JC, Gurley KA, Elliott SA, Sánchez Alvarado A. Planarian Hh signaling regulates regeneration polarity and links Hh pathway evolution to cilia. Science. $2009 \mathrm{Dec}$ 4;326(5958):1406-10.

20. Azimzadeh J, Wong ML, Downhour DM, Sánchez Alvarado A, Marshall WF. Centrosome loss in the evolution of planarians. Science. 2012 Jan 27;335(6067):461-3.

21. Arnold CP, Benham-Pyle BW, Lange JJ, Wood CJ, Sánchez Alvarado A. Wnt and TGF $\beta$ coordinate growth and patterning to regulate size-dependent behaviour. Nature. 2019 Aug;572(7771):655-9.

22. Liu S-Y, Selck C, Friedrich B, Lutz R, Vila-Farré M, Dahl A, et al. Reactivating head regrowth in a regeneration-deficient planarian species. Nature. 2013 Aug 1;500(7460):81-4.

23. Sikes JM, Newmark PA. Restoration of anterior regeneration in a planarian with limited regenerative ability. Nature. 2013 Aug 1;500(7460):77-80.

24. Umesono Y, Tasaki J, Nishimura Y, Hrouda M, Kawaguchi E, Yazawa S, et al. The molecular logic for planarian regeneration along the anterior-posterior axis. Nature. 2013 Jul 24;500:73.

25. Zhu SJ, Pearson BJ. (Neo)blast from the past: new insights into planarian stem cell lineages. Curr Opin Genet Dev. 2016 Oct;40:74-80.

26. Wagner DE, Wang IE, Reddien PW. Clonogenic neoblasts are pluripotent adult stem cells that underlie planarian regeneration. Science. 2011 May 13;332(6031):811-6. 
27. Eisenhoffer GT, Kang H, Sánchez Alvarado A. Molecular analysis of stem cells and their descendants during cell turnover and regeneration in the planarian Schmidtea mediterranea. Cell Stem Cell. 2008 Sep 11;3(3):327-39.

28. Almazan EMP, Lesko SL, Markey MP, Rouhana L. Girardia dorotocephala transcriptome sequence, assembly, and validation through characterization of piwi homologs and stem cell progeny markers. Dev Biol. 2018 Jan 15;433(2):433-47.

29. Reddien PW, Oviedo NJ, Jennings JR, Jenkin JC, Sánchez Alvarado A. SMEDWI-2 is a PIWIlike protein that regulates planarian stem cells. Science. 2005 Nov 25;310(5752):1327-30.

30. Guo L, Accorsi A, He S, Guerrero-Hernández C, Sivagnanam S, McKinney S, et al. An adaptable chromosome preparation methodology for use in invertebrate research organisms. BMC Biol. 2018 Feb 26;16(1):25.

31. Rozanski A, Moon H, Brandl H, Martín-Durán JM, Grohme MA, Hüttner K, et al. PlanMine 3.0-improvements to a mineable resource of flatworm biology and biodiversity [Internet]. Vol. 47, Nucleic Acids Research. 2019. p. D812-20. Available from: http://dx.doi.org/10.1093/nar/gky1070

32. Waterhouse RM, Seppey M, Simão FA, Manni M, loannidis P, Klioutchnikov G, et al. BUSCO Applications from Quality Assessments to Gene Prediction and Phylogenomics. Mol Biol Evol. 2018 Mar 1;35(3):543-8.

33. Swapna LS, Molinaro AM, Lindsay-Mosher N, Pearson BJ, Parkinson J. Comparative transcriptomic analyses and single-cell RNA sequencing of the freshwater planarian Schmidtea mediterranea identify major cell types and pathway conservation [Internet]. Vol. 19, Genome Biology. 2018. Available from: http://dx.doi.org/10.1186/s13059-018-1498-x

34. An Y, Kawaguchi A, Zhao C, Toyoda A, Sharifi-Zarchi A, Mousavi SA, et al. Draft genome of Dugesia japonica provides insights into conserved regulatory elements of the brain restriction gene nou-darake in planarians. Zoological Lett. 2018 Aug 29;4:24.

35. Grohme MA, Schloissnig S, Rozanski A, Pippel M, Young GR, Winkler S, et al. The genome of Schmidtea mediterranea and the evolution of core cellular mechanisms. Nature. 2018 Feb $1 ; 554(7690): 56-61$.

36. Guan D, McCarthy SA, Wood J, Howe K, Wang Y, Durbin R. Identifying and removing haplotypic duplication in primary genome assemblies. Bioinformatics. 2020 May 1;36(9):28968.

37. Kenk R. SEXUAL AND ASEXUAL REPRODUCTION IN EUPLANARIA TIGRINA (GIRARD). Biol Bull. 1937 Oct 1;73(2):280-94.

38. Grasso M. Further Observations on the Induction of Sexuality in Agamous Planarian Strains. Boll Zool. 1973 Jan 1;40(3-4):393-7.

39. Grasso M. Some Aspects of Sexuality and Agamy in Planarians. Boll Zool. 1974 Jan $1 ; 41(4): 379-93$.

40. Stocchino GA, Sluys R, Harrath AH, Mansour L, Manconi R. The invasive alien freshwater flatworm Girardia tigrina (Girard, 1850) (Platyhelminthes, Tricladida) in Western Europe: new insights into its morphology, karyology and reproductive biology. Contrib Zool. 2019 Jun 26;88(2):236-56. 
41. Cochet-Escartin O, Carter JA, Chakraverti-Wuerthwein M, Sinha J, Collins E-MS. Slo1 regulates ethanol-induced scrunching in freshwater planarians [Internet]. Vol. 13, Physical Biology. 2016. p. 055001. Available from: http://dx.doi.org/10.1088/1478-3975/13/5/055001

42. Schindelin J, Arganda-Carreras I, Frise E, Kaynig V, Longair M, Pietzsch T, et al. Fiji: an opensource platform for biological-image analysis. Nat Methods. 2012 Jun 28;9(7):676-82.

43. Jongebloed WL, Stokroos I, Van der Want JJ, Kalicharan D. Non-coating fixation techniques or redundancy of conductive coating, low kV FE-SEM operation and combined SEM/TEM of biological tissues. J Microsc. 1999 Feb;193(Pt 2):158-70.

44. Wang Y, Xu W, Maddera L, Tsuchiya D, Thomas N, Yu CR, et al. Alkaline phosphatase-based chromogenic and fluorescence detection method for BaseScope ${ }^{\mathrm{TM}}$ In Situ hybridization. J Histotechnol. 2019 Dec;42(4):193-201.

45. Jordan T, Williams D, Criswell S, Wang Y. Comparison of bleaching protocols utilizing hematoxylin and eosin stain and immunohistochemical proliferation marker MCM3 in pigmented melanomas. J Histotechnol. 2019 Dec;42(4):177-82.

46. King RS, Newmark PA. In situ hybridization protocol for enhanced detection of gene expression in the planarian Schmidtea mediterranea. BMC Dev Biol. 2013 Mar 12;13:8.

47. Pearson BJ, Eisenhoffer GT, Gurley KA, Rink JC, Miller DE, Sánchez Alvarado A. Formaldehyde-based whole-mount in situ hybridization method for planarians. Dev Dyn. $2009 ; 238(2): 443-50$.

48. Zeng A, Li H, Guo L, Gao X, McKinney S, Wang Y, et al. Prospectively Isolated Tetraspanin+ Neoblasts Are Adult Pluripotent Stem Cells Underlying Planaria Regeneration. Cell. 2018 Jun 14;173(7):1593-608.e20.

49. Grabherr MG, Haas BJ, Yassour M, Levin JZ, Thompson DA, Amit I, et al. Full-length transcriptome assembly from RNA-Seq data without a reference genome. Nat Biotechnol. 2011 May 15;29(7):644-52.

50. Chen Y-A, Lin C-C, Wang C-D, Wu H-B, Hwang P-I. An optimized procedure greatly improves EST vector contamination removal. BMC Genomics. 2007 Nov 13;8(1):416.

51. Haas BJ, Papanicolaou A, Yassour M, Grabherr M, Blood PD, Bowden J, et al. De novo transcript sequence reconstruction from RNA-seq using the Trinity platform for reference generation and analysis. Nat Protoc. 2013 Aug;8(8):1494-512.

52. Li W, Jaroszewski L, Godzik A. Clustering of highly homologous sequences to reduce the size of large protein databases. Bioinformatics. 2001 Mar;17(3):282-3.

53. Camacho C, Coulouris G, Avagyan V, Ma N, Papadopoulos J, Bealer K, et al. BLAST+: architecture and applications. BMC Bioinformatics. 2009 Dec 15;10:421.

54. Li L, Stoeckert CJ Jr, Roos DS. OrthoMCL: identification of ortholog groups for eukaryotic genomes. Genome Res. 2003 Sep;13(9):2178-89.

55. Edgar RC. MUSCLE: multiple sequence alignment with high accuracy and high throughput. Nucleic Acids Res. 2004 Mar 19;32(5):1792-7.

56. Capella-Gutiérrez S, Silla-Martínez JM, Gabaldón T. trimAl: a tool for automated alignment 
bioRxiv preprint doi: https://doi.org/10.1101/2020.07.01.183442; this version posted July 2, 2020. The copyright holder for this preprint (which

was not certified by peer review) is the author/funder, who has granted bioRxiv a license to display the preprint in perpetuity. It is made available under aCC-BY-NC-ND 4.0 International license.

trimming in large-scale phylogenetic analyses. Bioinformatics. 2009 Aug 1;25(15):1972-3.

57. Smith SA, Dunn CW. Phyutility: a phyloinformatics tool for trees, alignments and molecular data. Bioinformatics. 2008 Mar 1;24(5):715-6.

58. Kozlov AM, Darriba D, Flouri T, Morel B, Stamatakis A. RAxML-NG: a fast, scalable and userfriendly tool for maximum likelihood phylogenetic inference. Bioinformatics. 2019 Nov $1 ; 35(21): 4453-5$. 


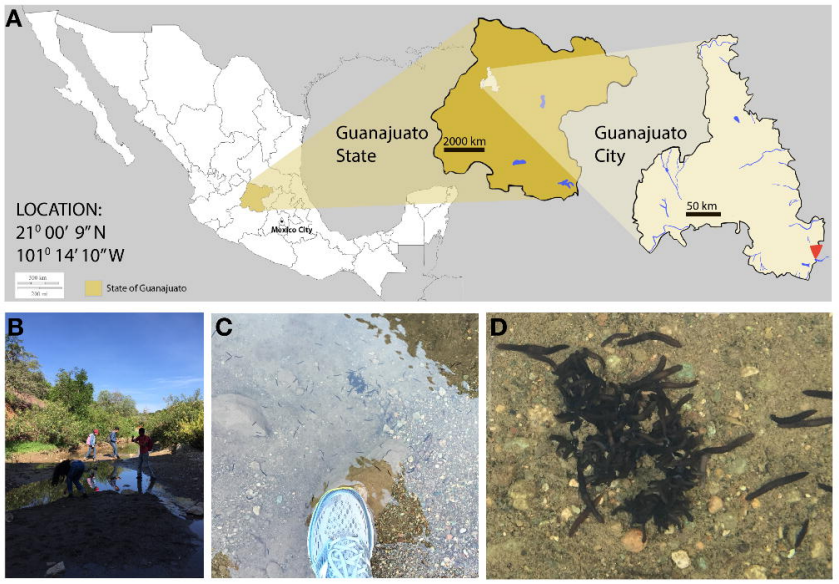




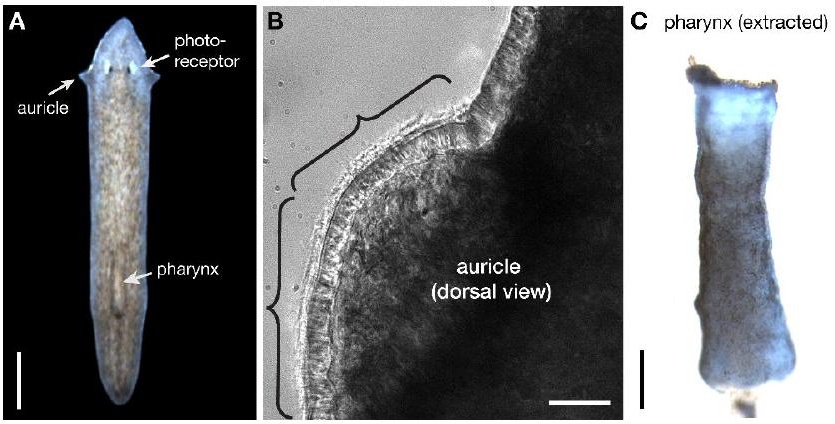




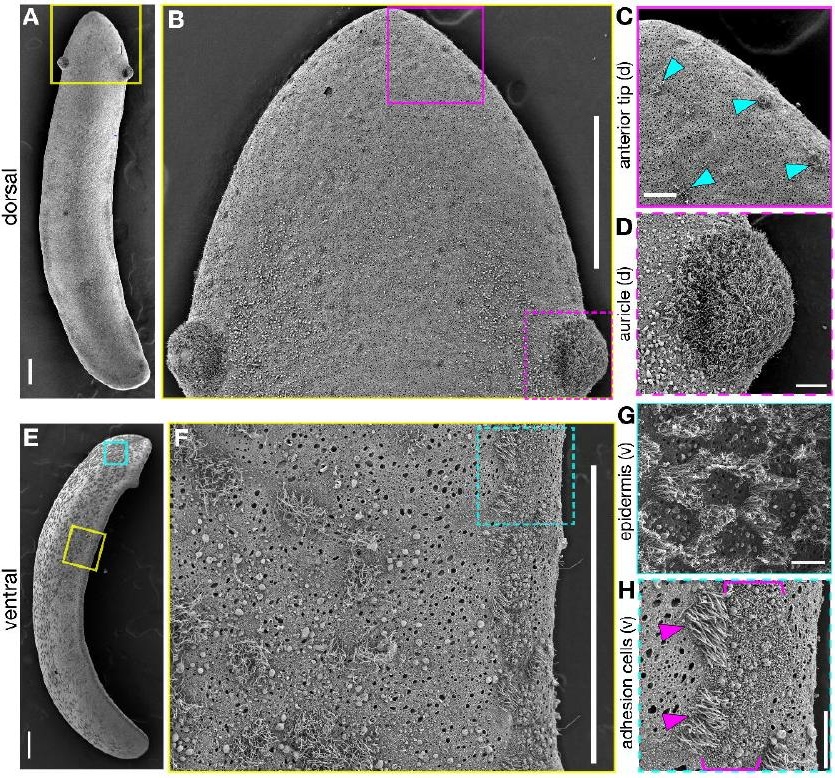




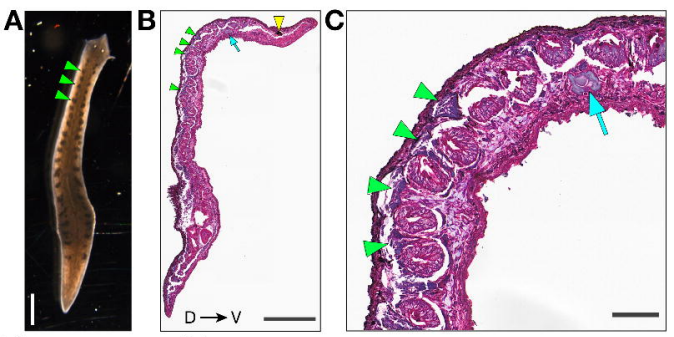

D

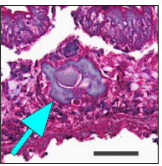

E

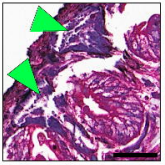

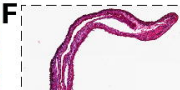

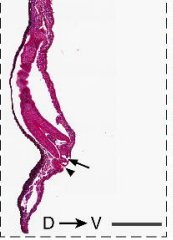

G

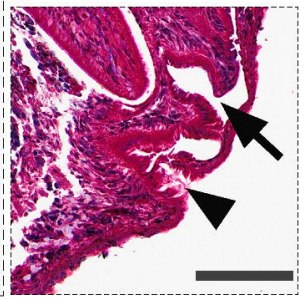




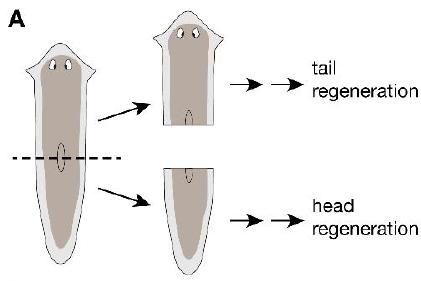

\section{B tail regeneration}

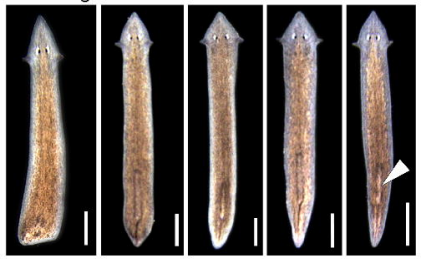

\section{C head regeneration}

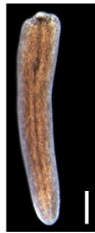

4 dpa

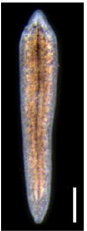

6 dpa

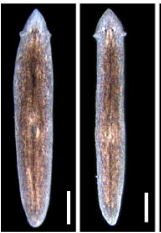

8 dpa

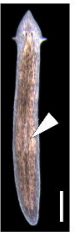

11 dpa 15 dpa 


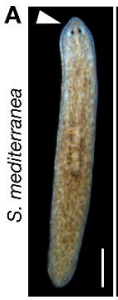

16 dpi

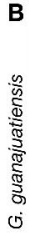

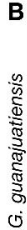

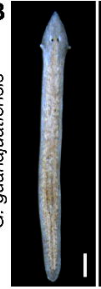

21dpi

28 dpi

35 dpi

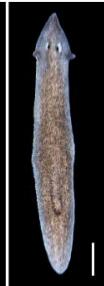

C

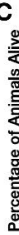

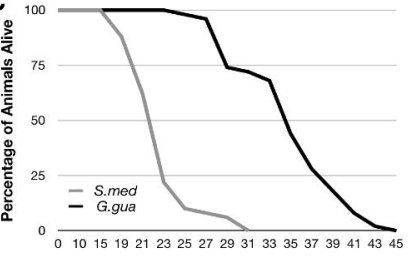

Days post $10 \mathrm{~K}$ rad Irradiation

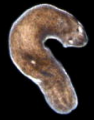

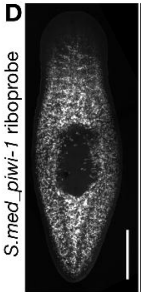
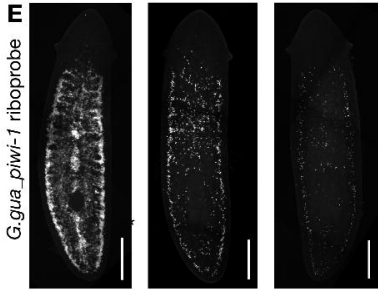

F

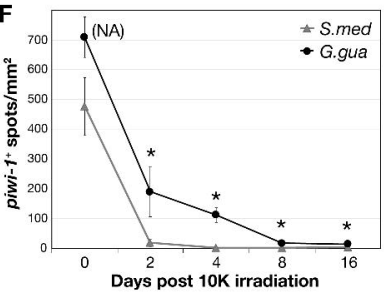




\section{D phylogenetic tree (transcriptome-based)}
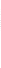

B nonredundant
peptides

peptides

transcripts

Missing (M) Complete (C) and Duplicated (D) Fragmented (F) Complete (C) and Single-copy (S) I

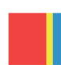

C:825 [S:781. D:44], F:22, M:131, n:978
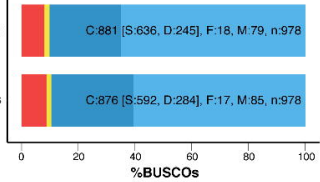

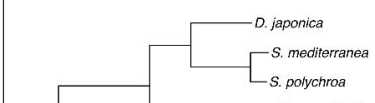

G. guanajuatiensis

G. dorotocephata D. lacteum assembled transcripts

\section{D. japonica \\ D. lacteum}

G. guanajuatiensis

P. nigra

P. tenuis

P. torva

S. mediterranea dd $v 6$

S. mediterranea SMEST

S. polychroa
C:874 [S:661, D:213], F:19, M:85, n:978

C:874 [S:650, D:224], F:34, M:70, n:978

C:876 [S:592, D:284], F:17, M:85, n:978

C:880 [S:677, D:203], F:15, M:83, п:978

C:867 [S:658, D:209], F:24, M:87, n:978

C:875 [S:650, D:225], F:18, M:85, n:978

C:875 [S:763, D:112], F:17, M:86, n:978

C.850 [S:588, D.262], F 25, M:103, n:978

C:871 [S:655, D:216], F:20, M:87, n:978

\begin{tabular}{|c|c|c|c|c|c|}
\hline 0 & 20 & 40 & 60 & 80 & 100 \\
\hline \multicolumn{6}{|c|}{$\%$ BUSCOs } \\
\hline
\end{tabular}

